\title{
Comparison of Sparse Representation and Fourier Discriminant Methods: Damage Location Classification in Indirect Lab-scale Bridge Structural Health Monitoring
}

\author{
Z. Wang ${ }^{1}$, S. Chen ${ }^{2}$, G. Lederman ${ }^{1}$, F. Cerda ${ }^{3}$, J. Bielak ${ }^{1}$, J. H.Garrett ${ }^{1}$, P. Rizzo ${ }^{4}$ and \\ J. Kovačević ${ }^{2}$
}

${ }^{1}$ Department of Civil and Environmental Engineering, ${ }^{2}$ Department of Electrical and Computer Engineering, Carnegie Mellon University, Pittsburgh, PA 15213, USA.

${ }^{3}$ Departamento de Ingeniería Civil, Universidad de Concepción, Concepción, 4070409, Chile.

${ }^{4}$ Department of Civil and Environmental Engineering, University of Pittsburgh, Pittsburgh, PA 15261, USA.

\begin{abstract}
This paper presents a novel method for interpreting data to improve the indirect structural health monitoring (SHM) of bridges. The research presented in the study is part of an ongoing study aimed at developing a novel SHM paradigm for the health assessment of bridges. In this paradigm, we envision the use of an instrumented vehicle that assesses a bridge's dynamic characteristics while traveling across the bridge. These characteristics are then correlated to the health of the structure by means of advanced signal processing and pattern recognition approaches. In this paper, we present and compare two classification algorithms that locate the presence of damages at well-defined locations on the structure: sparse representation and the Fourier discriminant methods, and find that the sparse representation method provides superior classification accuracy.
\end{abstract}

\section{INTRODUCTION}

The need for bridge structural health monitoring (SHM) has become clear in the aftermath of the I-35 Bridge collapse in Minneapolis. In the past two decades, the SHM community has done significant work to develop cost-effective technologies for the SHM of bridges and other large structures (Doebling et al. 1996; Chang 2011; Frangopol et al. 2010; Casciati and Giordano 2010). The majority of these works are based on direct methods, in which one or more arrays of sensors are mounted on the structure of interest to collect the dynamic and static characteristics of the bridge. These characteristics are then processed to infer the structure's health. The direct methods, however, may be too expensive to be implemented on an entire nation's bridge inventory. 
The indirect approach, on the other hand, overcomes this limit by installing sensors on a moving vehicle that, by crossing the bridge of interest, can capture the dynamic vehicle-bridge interaction and thus, indirectly, the dynamic characteristics of the bridge itself (Lin et al. 2005; Cerda et al. 2010). Using sophisticated signal processing algorithms, information about the bridge condition can be extracted from the vehicle-bridge interaction signals, which may allow for the detection and location of structural damage.

In this paper, we present the latest advancements from an ongoing project at Carnegie Mellon University and the University of Pittsburgh aiming to develop a holistic indirect health monitoring paradigm for bridges. In these experiments, we have focused on a laboratory-scale model, where we simulate the vehicle-structure interaction; the structure consists of one simplified laboratory-scaled bridge, inspected by a schematized vehicle traveling along rigid rails. The work presented in this paper follows (Cerda et al. 2013) and builds on it by studying a new feature-selection technique to more accurately identify and locate the presence of simulated defects induced on a bridge model. We compare Fourier discriminant method, which has been used in previous work, to the sparse representation method to detect and locate damages on the bridge.

\section{EXPERIMENTAL SETUP AND PROTOCOL}

Experimental setup. The model used in this study is shown in Figure 1; it consists of a mechanical system, a motion-control system, and a data-acquisition system. The mechanical system includes the structure and the car. The structure has an acceleration ramp, a bridge deck with two rails, and a deceleration ramp. The ramps are built to guarantee that the speed of the car is constant when traveling over the deck. The deck consists of a simply supported aluminum plate, $2438 \mathrm{~mm}$ ( 8 feet) long. Two angle beams act as girders, and two rails, mounted on the top surface, guide the car. The car, shown in Figure 2, has four independent suspension systems and four pedestal bases. Four accelerometers are secured to the car. Two sensors are mounted on top of the front and rear suspensions and two sensors are mounted on the top of the front and rear traverse stands. Hereafter, the sensors on the front are labeled as A.V. and the ones on the rear as B.V.

The vehicle is pulled across the bridge by a cable-and-belt system, which is connected to the motor, as indicated in Figure 1. The motion-control and the data-acquisition systems consist of a National Instruments ${ }^{\mathbb{B}}$ PXI system running in LabView $^{\circledR}$, a PXI Chassis (NI PXI 1031) with a motion-control card (NI PXI 7342), a motion interface (UMI 7772), a stepper drive (P70360) and a dual shaft stepper motor (NEMA 34). The acceleration data are collected at a $1667 \mathrm{~Hz}$ sampling rate and stored for data processing. More details about the experimental setup are reported in (Cerda et al. 2013). 


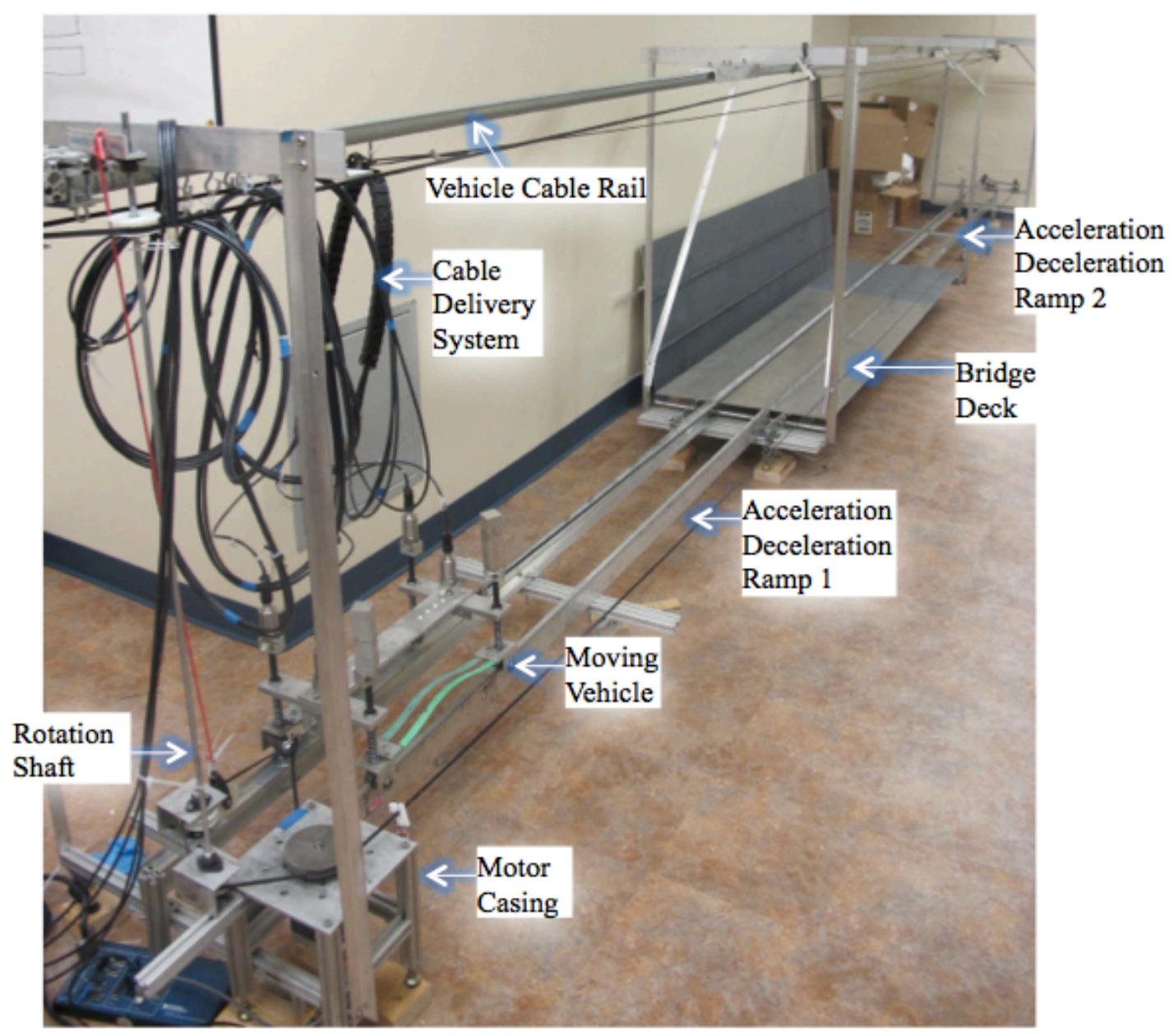

Figure 1. The lab-scale vehicle-bridge model.
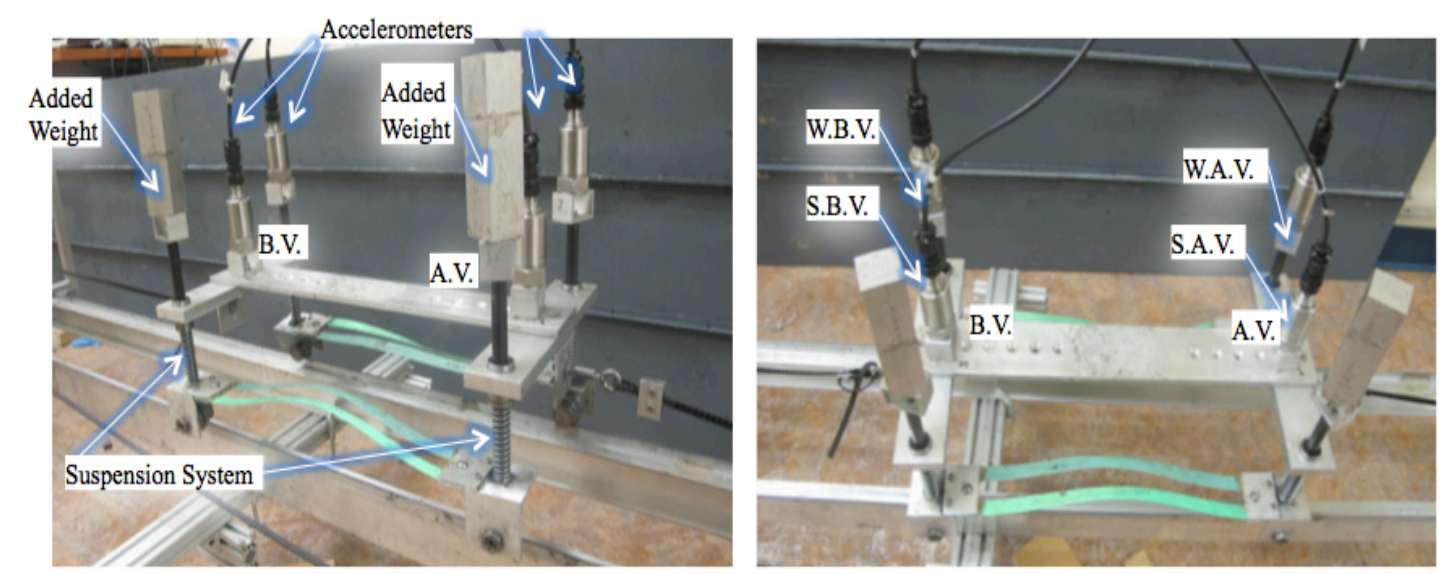

Figure 2. Close-up views of the vehicle. 

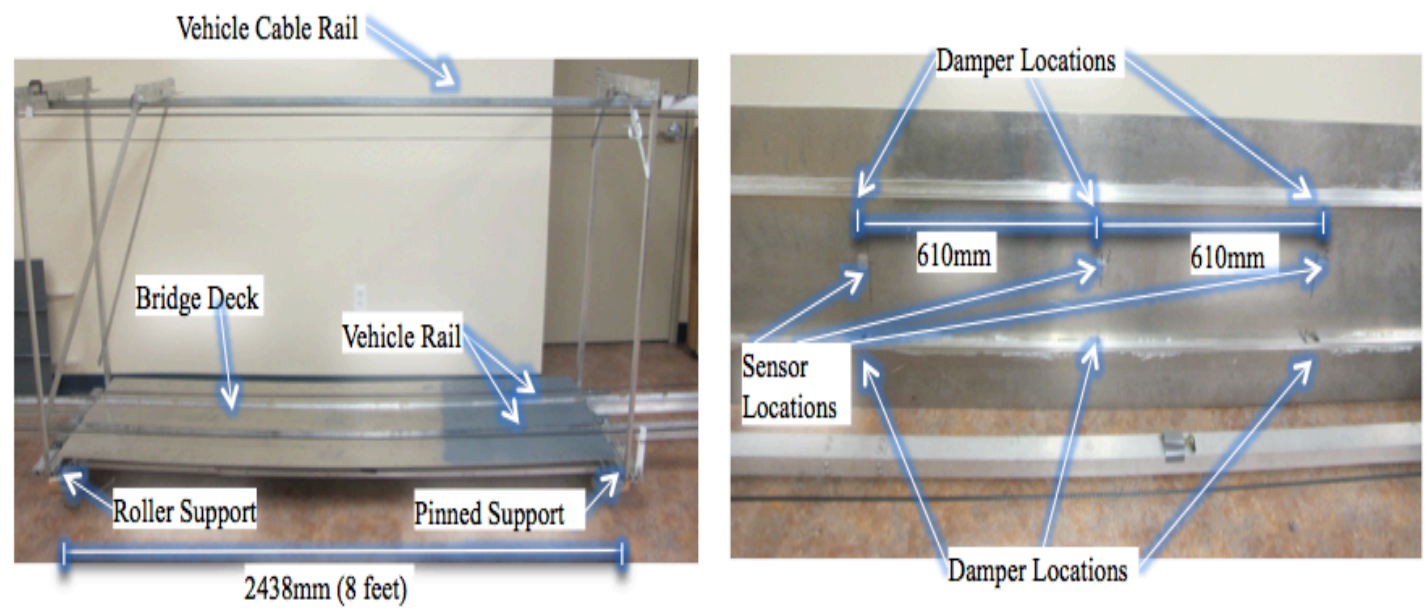

Figure 3. Close-up views of the bridge deck.

In addition to collecting data indirectly from the vehicle, we also collect data directly from the bridge itself to compare the two data collection techniques. Three sensors are mounted underneath the deck at the locations shown in Figure 3. They are located at $1 / 4 \mathrm{~L}, 1 / 2 \mathrm{~L}$ and $3 / 4 \mathrm{~L}$, where $\mathrm{L}$ is the deck's span. Figure 3 shows a close-up view of the deck.

Protocol. Damage is simulated by either placing mass, changing boundary restraints or installing dampers, each at four locations, on the bridge. Our aim is to find the accuracy with which we can classify the state of the bridge into these four categories (four locations). This four-class classification problem is repeated with two different masses, different rotational restraints and different damping conditions (see Table 1). The effects of these variations on the fundamental frequency and critical damping of the bridge are quantified by performing conventional free vibration tests. The results of these measurements are summarized in Table 1, which associates certain modal characteristics of the bridge to the simulated damage scenario. Each scenario is labeled to ease the identification of the damage. The first two numbers in the name string (scenario label) indicate a specific damage type and the last number the damage location, $0,1,2$ or 3 . Table 1 lists 24 scenarios. For each scenario, the car moved at 4 speeds, varying from $1.5 \mathrm{~m} / \mathrm{s}$ to $2.25 \mathrm{~m} / \mathrm{s}$ at $0.25 \mathrm{~m} / \mathrm{s}$. To assess repeatability, 32 measurements were taken. In total, we performed 24 (scenarios) x 4 (speeds) x 32 (iterations) $=3072$ trials.

Table 1. Damage scenarios and bridge characteristics.

\begin{tabular}{cccccc}
\hline & \multicolumn{5}{c}{ Bridge characteristics } \\
\hline Scenarios & SC- & Frequency $[\mathbf{H z}]$ & \% f shift & \% Critical damping & ${\text { \% d } \mathbf{d}_{\text {crit }} \text { shift }}$ \\
\hline & 020 & 7.86 & 1.06 & 15.63 & 0.20 \\
& 021 & 7.87 & 1.30 & 19.64 & 0.51
\end{tabular}




\begin{tabular}{|c|c|c|c|c|c|}
\hline hoo & 022 & 8.00 & 2.99 & 13.46 & -0.03 \\
\hline $\mathrm{Sol}_{2}$ & 023 & 8.17 & 5.20 & 24.85 & 0.91 \\
\hline$\because$ & 030 & 8.10 & 4.27 & 16.17 & -0.24 \\
\hline 단- & 031 & 8.30 & 4.27 & 21.05 & 0.61 \\
\hline$S_{0}$ & 032 & 8.40 & 8.14 & 19.53 & -0.5 \\
\hline 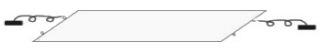 & 033 & 8.10 & 6.85 & 9.98 & -0.23 \\
\hline & 070 & 7.77 & 0.00 & 18.93 & 0.45 \\
\hline & 071 & 7.77 & 0.00 & 27.06 & 1.08 \\
\hline & 072 & 7.77 & 0.00 & 18.54 & -0.42 \\
\hline & 073 & 7.77 & 0.00 & 23.40 & 0.79 \\
\hline & 080 & 7.67 & -1.30 & 34.52 & 1.65 \\
\hline & 081 & 7.67 & -1.30 & 33.80 & 1.59 \\
\hline 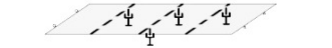 & 082 & 7.67 & -1.30 & 35.51 & 1.72 \\
\hline & 083 & 7.70 & -0.88 & 37.03 & 1.84 \\
\hline$\frac{10090}{\int_{1 / 2 L}} f_{1 / 2} w$ & 110 & 7.67 & -1.30 & 11.82 & -0.09 \\
\hline$\sigma_{100 g}^{1 / 2 \mathrm{~W}}$ & 111 & 7.67 & -1.30 & 12.28 & -0.06 \\
\hline $1009 \underset{1 / 4 \mathrm{~L}}{\rightleftarrows} / 1 \times 2 w$ & 112 & 7.67 & -1.30 & 11.70 & -0.10 \\
\hline $100 \mathrm{~g} \overbrace{}^{1 / 2 L} / 1 / 8 \mathrm{~W}$ & 113 & 7.67 & -1.30 & 13.81 & 0.06 \\
\hline 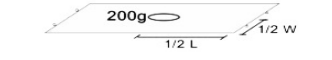 & 120 & 7.60 & -2.16 & 11.25 & -0.14 \\
\hline${ }_{1 / 2}^{\frac{W}{1 / 4} L}=200 \mathrm{~g}$ & 121 & 7.70 & -0.88 & 12.55 & -0.04 \\
\hline  & 122 & 7.77 & 0.00 & 12.62 & -0.03 \\
\hline $200 \mathrm{~g} \overbrace{1 / 2 \mathrm{~L}}^{1 / 8 \mathrm{w}}$ & 123 & 7.60 & -2.16 & 11.28 & -0.14 \\
\hline
\end{tabular}

\section{SIGNAL ANALYSIS AND CLASSIFICATION}

The task of distinguishing various bridge conditions can be treated as a signal classification problem that requires associating an input signal with a pre-defined class label. In this paper, we tested two classification algorithms: Fourier discriminant method and sparse representation method. Fourier discriminant method searches for the most discriminative frequency features and then uses support vector machine (SVM) (Duda et al. 2000) to classify those features. Sparse representation method searches for a dictionary to represent each class and assigns the signal to the class whose corresponding dictionary gives the minimum representation error. We now discuss these in more detail. 
(1) Classification based on Fourier discriminant method. The basic idea of this algorithm is to extract the most discriminative features of the vibrational signal in the frequency domain based on their discriminative power, and use those features to discriminate among different scenarios. Algorithm 1 as shown below summarizes the process. In the training phase, we first compute the discrete Fourier transform (DFT) of each signal in the training dataset. Then, we compute the discriminative power of each Fourier basis vector by using Fourier discriminant basis vector selection (Cerda et al. 2013) and recorde the order of the discriminative power. The $\mathrm{k}$ most discriminative Fourier basis vectors are then used as features. We then train an SVM classifier by using these features and their corresponding known labels. In the testing phase, we compute the DFT of each signal in the testing dataset, and then the Fourier discriminant features by retrieving the order of the discriminative power learned in the training phase. These features are then classified using the SVM classifier trained previously. More details can be found in (Cerda et al. 2013).

\section{Algorithm 1 (Fourier discriminant method) \\ Input: labeled training dataset. Output: class labels. \\ Training phase}

1. Compute the DFT of each signal in the training dataset.

2. Compute the discriminative power of each Fourier basis vector by using Fourier discriminant basis vector selection and record the order of the discriminative power.

3. Use $k$ most discriminative Fourier basis vectors as features

4. Train SVM classifier by using features from Step 3 and their corresponding known labels.

\section{Testing phase}

1. Compute the DFT of each signal in the testing dataset.

2. Compute the Fourier discriminant features by retrieving the order of the discriminative power that learns in the training phase.

3. Classify the features by using SVM classifier trained previously.

(2) Classification based on sparse representation method. Sparse representations have been shown to be a strong tool for representing and denoising signals (Wright et al. 2009). Although a signal may have high dimensionality, it could have a compact representation in a low-dimensional space, which means the signal can be sparsely represented without losing information. The motivation to use sparse representations when analyzing vibrational signals is that we assume there are only a few key factors influencing a vehicle-bridge system. By squeezing most information into a small number of elementary signals, we find these key factors. 
In sparse representations, signals can be represented by using a few expansion coefficients in a fixed over-complete dictionary, called sparse coding. The representation dictionary is updated by fitting the data, and usually referred to as dictionary learning. Each dictionary corresponds to a specific vehicle-bridge system. This classification can be done by labeling each signal and representing it by a dictionary that gives the minimum representation error. We now explain these concepts in more detail.

Sparse coding. Sparse coding computes the expansion coefficients based on the given signal and the dictionary. The exact solution is known as a nondeterministic polynomial hard problem, and thus, approximate algorithms are considered instead. Here, we use the orthogonal matching pursuit (OMP) algorithm (Chen et al. 1989). OMP is a greedy algorithm that selects the basis vector from a dictionary sequentially. In each iteration, the dictionary finds the best basis vector to fit the given signal and leaves the residue for the next iteration. Although it cannot provide a global optimum, it only involves the computation of inner products between the signal and basis vector, leading to a computationally efficient algorithm.

\section{Algorithm 2 (Sparse representation) \\ Input: labeled training dataset. Output: class labels. \\ Training phase \\ 1. Initialize a dictionary for each class and use OMP to represent the each signal in the training dataset. \\ 2. For each class, update the dictionary to fit to training signals by using K-SVD. \\ 3. Iterate Steps $1 \& 2$ in turn until the reconstruction error is below a given threshold and record the dictionaries for all classes. \\ Testing phase \\ 1. Represent each signal in the testing dataset on the dictionaries for all classes by using OMP. \\ 2. Label each signal to the class that the corresponding dictionary gives the minimum representation error.}

Dictionary learning. Dictionary learning updates the dictionary iteratively to reach the goal of expanding the given signal sparsely. Unlike traditional transforms, such as Fourier and wavelets, the dictionary in sparse representation is flexible and can be learned by fitting itself to the given signals. Some state-of-the-art algorithms are methods of optimal directions (MOD) (Engan et al. 1999) and K-SVD (Aharon et al. 
2006), which we use in this paper. It is designed to be a generalization of the K-means algorithm (MacQueen and J. B. 1967) and work towards minimizing an overall objective function.

We perform sparse coding and dictionary learning to get the dictionary and the representation coefficients for the given signals. Each signal is then assigned to the class for which the corresponding dictionary provides the minimum representation error. This algorithm is summarized in Algorithm 2.

\section{CLASSIFICATION RESULTS AND DISCUSSION}

We set up a similar damage location classification experiment in our previous work (Cerda 2012). To make the experiments more realistic, we lower the training ratio (the ratio of the number of the training samples and all samples) from $95 \%$ to $50 \%$. Figures 4 and 5 shows the accuracies of classifying damage location for six different types of damage clusters, SC02-, SC03-, SC07-, SC08-, SC11- and SC12-, for the Fourier discriminant method and sparse representation method, respectively. Each type of a damage cluster has four different location scenarios indicated by the last number in the name string as in Table 1. The accuracies in the figures are the averaged accuracies over four speeds and all iterations. The red vertical bar represents the corresponding standard deviation. We show results associated with the transducers mounted on the bridge and the sensors mounted on the car's suspensions and wheels. On average, the sparse representation method provides about $10 \%$ higher accuracy than the Fourier discriminant method. The lowest accuracies for both methods are for SC08-; from Table 1, we see that the difference among the four scenarios in SC08- is only changing the locations of the two dampers, and only one out of four scenarios changes the frequency of the vibration by a small amount. Thus, these variations cannot be detected easily. Even then, sparse representation method is still able to improve accuracy by about $10 \%$ compared to Fourier discriminant method. Overall, we conclude that by using the sparse representation method, most of the accuracies are above $70 \%$ and all of them are above $60 \%$.

We also evaluated the effect of speed on the classification accuracies of the two algorithms. Figure 6 shows the classification accuracy of both algorithms as a function of the vehicle speed. Two interesting trends are visible: (1) We see higher classification accuracy when using sparse representations as previously noted. (2) We observe a decrease in the classification accuracy as the speed increases. This accuracy decrease can be explained as follows: as the speed goes up, the length of the analyzed signal is shorter and therefore less information from the bridge can be extracted. Also, as the excitation frequency increases, the vehicle may excite higher modes that may be less sensitive to the presence of the simulated damage. 


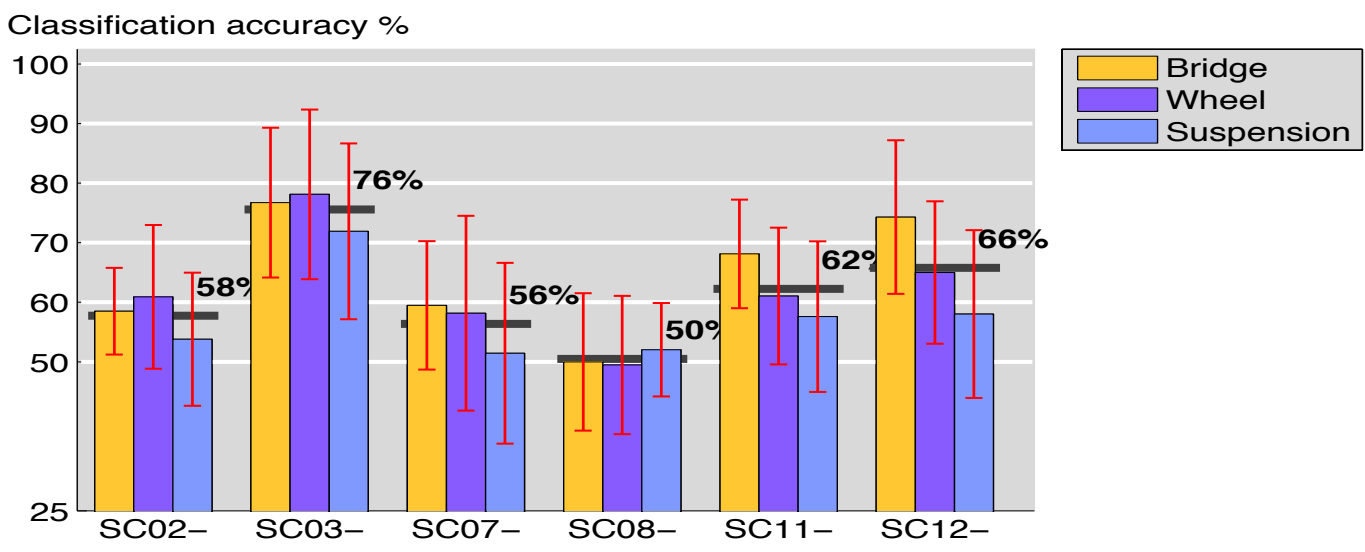

Figure 4. Classification accuracy using Fourier discriminant method.


Figure 5. Classification accuracy using sparse representation method.

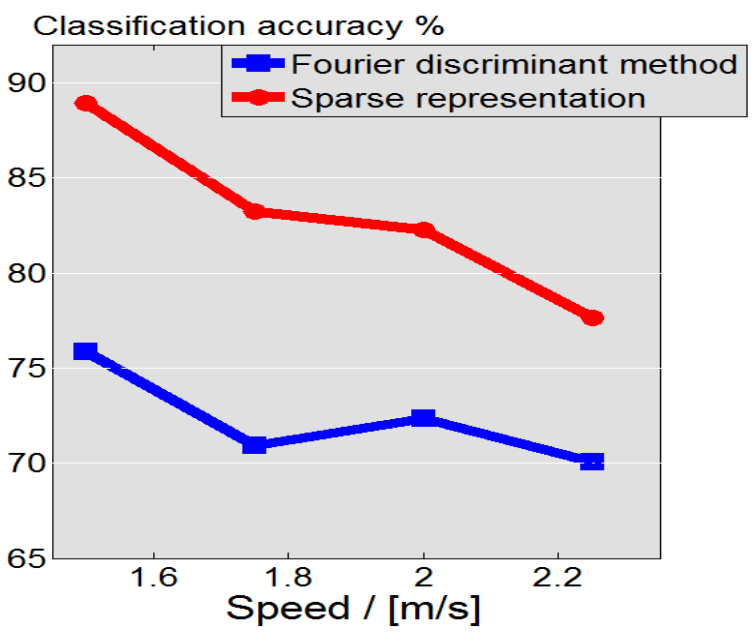

Figure 6. Classification accuracy as a function of speed. 


\section{CONCLUSIONS}

We presented the latest advancement of an ongoing project at Carnegie Mellon University and at the University of Pittsburgh that aims at developing a holistic approach for the indirect health monitoring of bridges. We compared a proposed novel classification algorithm with a previous method (Cerda 2012). The proposed method, using sparse representations, was tested on vibrational signals collected from a laboratory-scale bridge model, which simulates the vehicle-bridge interaction. The results are compared to Fourier discriminant method that has been used previously. We found that the sparse representations allows for higher classification accuracy when compared with the Fourier discriminant method, and that the accuracy of each algorithm is inversely proportional to the speed of the car.

\section{ACKNOWLEDGEMENTS}

The authors gratefully acknowledge the support of U.S. National Science Foundation awards 1130616 and 1017278 and of a University Transportation Center grant (DTRT12-G-UTC11) from the US Department of Transportation.

\section{REFERENCES}

Aharon, M., Elad, M. and Bruckstein, A. (2006). "The K-SVD: An Algorithm for Designing Overcomplete Dictionaries for Sparse Representation". IEEE Transactions on Signal Processing, vol. 54, no. 11: 4311 -4322. doi: 10. 1109/ TSP. 2006. 881199.

Casciati, F., and Giordano, M. (2010). Structural Health Monitoring 2010: Proceedings of the Fifth European Workshop on Structural Health Monitoring, Sorrento, Italy. DEStech Publications, Inc.

Cerda, F., Garrett, J., Bielak, J., Bhagavatula, R. and Kovačević, J. (2010). "Exploring Indirect Vehicle-Bridge interaction for SHM". In Proc. of the Fifth International Conference on Bridge Maintenance, Safety and Management, IABMAS2010, Philadelphia, USA, 696-702.

Cerda, F. (2012). Indirect Structural health Monitoring of Bridges: Mathematical Models and Laboratory Experiments. Ph.D Dissertation, Carnegie Mellon University. Pittsburgh, PA.

Cerda, F., Chen, S., Bielak, J., Garrett, J., Rizzo, P., Kovačević, J. (2013). “Indirect Structural Health Monitoring Of A Simplified Laboratory-scale Bridge Model". Submitted to Smart Structures and Systems (Special Issue: Challenge on bridge health monitoring utilizing vehicle-induced vibrations.), in review. 
Chang, F. (2011). Structural Health Monitoring 2011: Condition-Based Maintenance and Intelligent Structures. Proceedings of the Eighth International Workshop on Structural Health Monitoring. DEStech Pub.

Chen, S., Billings, S. A., and Luo, W. (1989). "Orthogonal Least Squares Methods And Their Application to Non-linear System Identification". Int. J. Contr., vol. 50, no. 5, pp. 1873-96.

Doebling, S. W., Farrar, C. R., Prime, M. B., Shevitz, D. W. (1996). "Damage Identification and Health Monitoring of Structural and Mechanical Systems From Changes in Their Vibration Characteristics: A Literature Review". Los Alamos National Laboratory Report. Report Number: LA-13070-MS.

Duda, R., Hart, P., and Stork, D. (2000). Pattern Classification (2nd Edition). Wiley-Interscience.

Engan, K., Aase, S. O., and Hakon-Husoy, J. H. (1999). "Method of Optimal Directions for Frame Design". In Proc. IEEE Int. Conf. Acoust., Speech, Signal Process., vol. 5, pp. 2443-2446.

Frangopol, D., Sause, R., and Kusko, C. S. (2010). Bridge Maintenance, Safety and Management - IABMAS'10: in Proc. of the Fifth International IABMAS Conference (1st Edition). CRC Press. Philadelphia, USA.

Lin, C.W., and Yang, Y.B. (2005). "Use of a passing vehicle to scan the fundamental bridge frequencies: An experimental verification". Engineering Structures 27, no. 13 (November): 1865-1878.

Macqueen, J. B. (1967). "Some Methods for Classification and Analysis of Multivariate Observations". 1:281-297. University of California Press.

Wright, J., Yang, A. Y., Ganesh, A., Sastry, S. S. and Ma, Y. "Robust Face Recognition via Sparse Representation." IEEE Transactions on Pattern Analysis and Machine Intelligence. Vol. 31, no. 2: 210-227. doi: 10. 1109/ TPAMI. 2008. 79. 